\section{El nuevo modelo de atención imagine y su efecto en la satisfacción de los clientes del Banco Interbank de la tienda Comas 138, año 2015 - 2017}

\author{
The new imagine attention model and its effect on the \\ customer satisfaction of the Interbank Bank of the Comas \\ store 138, year 2015 - 2017
}

\begin{abstract}
RESUMEN
La presente investigación está enfocada en poder demostrar que el nuevo modelo de atención Imagine tiene un efecto en la satisfacción de los clientes del Banco Interbank de la tienda Comas 138, con el propósito de comprobar la aceptación de este nuevo modelo de atención. En ese sentido la investigación es de tipo aplicativa puesto que todos sus aspectos son teorizados, aunque sus alcances son prácticos, asimismo el diseño es correlacional ya que su finalidad es describir la relación de las variables y examinar su repercusión. La obtención de los datos fue a través de encuestas realizadas a una muestra de 277 clientes. Por lo consiguiente se realizó la medición y el análisis de la fiabilidad del coeficiente de correlación, así como la puntuación del Alfa de Cronbach obteniendo como resultado 0.97, por lo cual se determina que existe muy buena correlación entre el nuevo modelo de atención Imagine y su efecto en la satisfacción de los clientes del Banco Interbank de la tienda Comas 138. En síntesis, resulta cierto decir que el nuevo modelo de atención Imagine si tiene un efecto en la satisfacción de los clientes del banco Interbank de la tienda Comas 138.
\end{abstract}

Palabras claves: Formato Imagine; omnicanalidad; design thinking; gestión del cliente; canales alternativos de atención.

\begin{abstract}
The present research is focused on being able to demonstrate that the new Imagine service model has an effect on the satisfaction of the Interbank Bank customers of the Comas 138 store, in order to verify the acceptance of this new service model. In this sense, the research is of an applicative type since all its aspects are theorized, although its scope is practical, also the design is correlational since its purpose is to describe the relationship of the variables and examine their impact. The data was obtained through surveys carried out on a sample of 277 clients. Therefore, the measurement and analysis of the reliability of the correlation coefficient was carried out, as well as the Cronbach's Alpha score, obtaining 0.97 as a result, which is why it is determined that there is a very good correlation between the new Imagine care
\end{abstract}

Luis Enrique Cauti Escobar cauti_24@hotmail.com Universidad Científica del Sur

Presentado: 03/03/2020 - Aceptado: 01/10/2020 - Publicado: 26/11/2020

(C) Los autores. Este artículo es publicado por Gestión en el Tercer Milenio de la Facultad de Ciencias Administrativas de la Universidad Nacional Mayor de San Marcos. Este es un artículo de acceso abierto, distribuido bajo los términos de la licencia Creative Commons Atribucion - No Comercia_Compartir Igual 4.0 Internacional. (http://creativecommons.org/licenses/by-nc-sa/4.0/) que permite el uso no comercial, distribución y reproducción en cualquier medio, siempre que la obra original sea debidamente citada. 
model and its effect on the satisfaction of the Interbank Bank customers of the Comas 138 store. In summary, it is true to say that the new service model Imagine if it has an effect on the satisfaction of the Interbank bank customers of the Comas 138 store.

Keywords: Imagine format; omnichannel; design thinking; customer management; alternative care channels.

\section{INTRODUCCIÓN}

Hoy en día cuando un cliente tiene la necesidad de elegir entre varias empresas ese sentimiento profundo de aprobación es tan eficaz en el proceso de persuasión que lo lleva a comprar en una empresa determinada y no a otra, por esto los bancos también saben que están obligados a fidelizar a sus clientes más que nunca, dado a que los precios y servicios bancarios son, en términos generales, los mismos y saben también que el servicio es la clave para conservar el negocio y para tener una diferenciación (Franzmeier, 1994); dentro de este orden de ideas el factor calidad es actualmente uno de los ítems principales para poder atender y satisfacer las necesidades y expectativas de los clientes en la adquisición de productos y servicios. Hoy por hoy vemos agresivas disputas entre las organizaciones para ser la primera opción de compra, esta tendencia no solo se observa en el sector bancario sino también en las demás industrias, pero la implementación para lograr una mejora en la calidad de atención al cliente puede llegar a tardar un tiempo prolongado debido a que el proceso de fidelización de los clientes no es una tarea de corto plazo. Por lo consiguiente, Solorzano y Aceves (2013) consideran que en la actualidad la fidelización de los clientes es muy difícil de conseguir ya que ellos antes de adquirir productos o servicios realizan frecuentes comparaciones para poder recabar información de los principales beneficios, costos y penalidades para la adquisición de productos. Entonces el cliente que no encuentra una atención de calidad puede tomar la decisión de cambiar productos y empresas las veces que ellos crean necesario y esto puede producir que las políticas de servicio al cliente establecidas queden relegadas. Por lo expuesto, las instituciones deben tener muy claro su propósito para ejecutar el cumplimento de sus políticas y procesos para atender la satisfacción de sus clientes, preocuparse por sus necesidades reales para que se sientan muy valorados y no migrar a hacia los competidores.

Diversas investigaciones muestran una fuerte tendencia en la aparición de canales alternativos de atención, los clientes pueden realizar distintas transacciones financieras sin necesidad de ir a las oficinas del banco. Es principalmente por este motivo que la estrategia en la penetración de la banca digital viene desarrollándose con más rapidez en los últimos años migrando la estrategia de atención tradicional hacia canales de atención omnicanal. Ya no es suficiente para un banco ofrecer sus productos o servicios por el canal tradicional, ya que esto representa tener una gran desventaja frente a sus competidores debido a que la gran mayoría cuentan con la capacidad de atender a sus clientes por medios omnicanales.

La capacidad que tienen los bancos para poder adaptarse a esta realidad permite que sus clientes y usuarios puedan ser atendidos de manera ágil, en este sentido se comprende que cada cliente tiene distintas necesidades y esta nueva tendencia impulsa a los bancos a tener una visión integral de sus clientes para que de esta manera puedan satisfacer a la medida sus necesidades y expectativas. La estrategia omnicanal no solo se debe centrar en la atención en términos de accesibilidad, sino que deben también tener la capacidad de solucionar las necesidades de los clientes y que la interacción con estos múltiples canales de atención sea de fácil uso (Vieyra, 2016). Esta reciente tendencia sumada a la perspectiva de los clientes simboliza un cambio disruptor que genera un alto impacto y por esta razón los bancos se ven forzados a monitorear de manera constante el entorno del cliente asegurándose que las herramientas de la banca digital posibiliten obtener un alto tráfico de información y datos que permitan en un futuro cercano concretarse en transacciones rentables y de alto valor. 
La industria financiera ha venido cambian- entre la variable independiente "el nuevo modedo la manera tradicional de manejar su negocio. A lo largo del último decenio, el fortalecimiento, el mundo global, las estrictas normas impuestas por los entes reguladores financieros y las constantes variaciones en la demanda de los clientes han trastocado dicho sector y a consecuencia de esto las instituciones tiene disputas con otras que ya se encuentran muy afianzadas en este sector (Riveroll, 2016). Para poder tener cada vez mayor market share y esto lo hacen a través de innovaciones constantes, agilidad en la atención, transparencia en la información y quizá lo más importante un servicio de calidad a los clientes.

Ante la gran necesidad de atender a los clientes de una forma innovadora y con altos estándares de calidad en el servicio, el banco Interbank presentó el año 2013 la primera tienda en el Perú con el modelo de atención Imagine la cual se ubicada en el centro comercial Real Plaza Centro Cívico (Interbank, 2017). Este formato vanguardista es actualmente único en nuestro país el cual fue implementado por la multinacional Ideo que es una empresa de diseño global y consultora líder en temas de diseño de productos, servicios, entornos y experiencias digitales a nivel mundial. Los principales atributos de este nuevo formato son:

Cero colas físicas, el cliente es registrado con su documento de identidad en una plataforma digital y puede tomar asiento degustando un café gratis mientras espera para su atención. Zona de aprendizaje financiero, aquí el cliente puede navegar por internet y revisar cómodamente las principales condiciones de los productos y servicios que ofrece el banco (Uccelli, 2013). Menos obstáculos físicos, las repisas que se usan en los counters son más amplias y abiertas para así tener una interacción más cómoda con el cliente. Promover los beneficios de utilizar los medios de atención alternos, como la banca electrónica, banca digital y banca telefónica. Notificaciones por mensajes de texto, cuando el cliente es registrado inicialmente en el sistema de colas, registra su número de celular para que sea notificado minutos antes de ser atendido.

\section{MÉTODOS}

El tipo de investigación es correlacional ya que su finalidad es describir la relación que existe lo de atención Imagine" y la variable dependiente "satisfacción del cliente banco Interbank" y poder analizar su incidencia e interrelación. El alcance es descriptivo, ya que tiene como propósito investigar la incidencia y los valores en que se manifiestan las variables detalladas en nuestra investigación para poder resolver el problema.

La población de nuestra investigación es de 980 clientes que se están sectorizados con la tienda Comas 138, son personas naturales dependiente e independientes y que tiene productos activos, pasivos y fondos de inversión. Fueron encuestados mediante un cuestionario de elaboración propia que mide las dimensiones de la variable independiente y dependiente. Se determinó un tipo de muestra probabilística y aleatoriamente, el volumen de nuestra muestra de nuestra investigación corresponde a un total de 277 clientes de los cuales $43 \%$ son mujeres (119) y 57\% son hombres (158) quienes fueron entrevistados dentro de las instalaciones del banco. Se utilizó un margen de error del $5 \%$, un nivel de confianza del $95 \%$ y una población de 980 clientes, entonces para cuantificar el tamaño de muestra (n) tenemos la siguiente ecuación:

$$
\begin{aligned}
& \mathrm{z}^{\wedge} 2\left(\mathrm{p}^{*} \mathrm{q}\right) \\
& \mathrm{n}=\quad \mathrm{e}^{\wedge} 2+\left(\mathrm{z}^{\wedge} 2\left(\mathrm{p}^{*} \mathrm{q}\right)\right) \\
& \text { N }
\end{aligned}
$$

Vemos que:

z: Nivel de confianza 95\%

N: Proporción de la población 980 clientes

p: Posibilidad de logro 0.5

q: Posibilidad de fracasar 0.5

e: Error $5 \%$

Entonces realizando el cálculo hallamos que el tamaño de la muestra es $n=277$

\section{RESULTADOS}

Los resultados comprueban que el nuevo modelo de atención Imagine tiene un efecto representativo en la satisfacción de los clientes del 
banco Interbank de la tienda Comas 138, fueron corroborados con las pruebas de hipótesis chi-cuadrado reflejados en las tablas de contingencia lo que dieron a conocer la relación de las variables de nuestras hipótesis. Se usó la herramienta estadística Alpha de Cronbach para ratificar su medición y también el software SPSS versión 25 para medir la fiabilidad obteniendo una puntuación de 0.972 , lo cual nos indica que existe una muy buena correlación por lo que se determina que nuestra herramienta cumple con la condición correcta de recopilación de los datos de nuestra investigación. Véase Tabla 1.

Tabla 1

Resultado de la fiabilidad

\begin{tabular}{cc}
\multicolumn{2}{c}{ Estadística de fiabilidad } \\
Alfa de Cronbach & N de elementos \\
0,972 & 42 \\
\hline
\end{tabular}

Nota: Elaboración propia a partir de la aplicación del software SPSS 2019 versión 25.

Se realizó el análisis del producto recabado de las dimensiones de la variable dependiente, estos indican una alta satisfacción de los clientes respecto a la transparencia en la información de los funcionarios del banco Interbank. En la Tabla 2, podemos notar que existe relación entre la transparencia de la información y el nuevo formato Imagine debido a que 147 clientes opinan alta satisfacción mientras que 88 valoran buena, de un total de 277 encuestados.

Finalmente se calculó el Chi-cuadrado de Pearson obteniendo como resultado $\left(\mathrm{X}^{2}=\right.$ 179.618) y dado que es mayor que al chi-cuadrado crítico $\left(\mathrm{X}_{\mathrm{cr}}=26.29\right)$, rechazamos nuestra hipótesis nula $\left(\mathrm{H}_{0}\right)$ y aprobamos la hipótesis alterna $\left(\mathrm{H}_{1}\right)$, se concluye que existe efecto significativo entre el nuevo modelo de atención Imagine y la satisfacción de los clientes. Véase Tabla 3.

La afinidad que tienen los funcionarios es trascendental en la atención al cliente. En la Tabla 4 se aprecia que los clientes están muy satisfechos respecto a la empatía de los colaboradores al momento de la atención, podemos notar que existe relación entre la empatía de los colaboradores y el nuevo modelo de atención Imagine puesto que 45 clientes tienen muy alta satisfacción mientras que 182 valoran alta satisfacción, de un total de 277 encuestados.

Finalmente se calculó el Chi-cuadrado de Pearson obteniendo como resultado (X2 = 229.387) y dado que es mayor que al chi-cuadrado crítico $(\mathrm{X} 2 \mathrm{cr}=21.02)$, rechazamos nuestra hipótesis nula (H0) y aprobamos la

Tabla 2

Congruencia entre la transparencia de información y el nuevo modelo de atención Imagine

\begin{tabular}{rlcccc} 
Nuevo modelo de atención & \multicolumn{1}{c}{$\begin{array}{c}\text { Consideración } \\
\text { del cliente }\end{array}$} & $\begin{array}{c}\text { Frecuencia } \\
\text { Baja }\end{array}$ & $\begin{array}{c}\text { Porcentaje } \\
\text { válido }\end{array}$ & $\begin{array}{c}\text { Porcentaje } \\
\text { Acumulado }\end{array}$ \\
& Regular & 22 & 7.9 & 7.9 \\
Transparencia de información & Buena & 10 & 3.6 & 11.6 \\
& Alta & 88 & 31.8 & 43.3 \\
& Muy alta & 147 & 53.1 & 96.4 \\
& Total & 277 & 100 & 100.0 \\
\hline
\end{tabular}

Nota: Elaboración propia a partir de la aplicación del software SPSS 2019 versión 25.

Tabla 3

Chi-cuadrado entre la transparencia de información y el nuevo modelo de atención Imagine

\begin{tabular}{lccc} 
& Valor & Df & Significación Asintótica \\
Chi-cuadrado de Pearson & 179,618 & 12 & .000 \\
Razón de verosimilitud & 105,012 & 12 & .000 \\
Asociación lineal por lineal & 85,566 & 1 & .000 \\
Número de casos válidos & 277 & & \\
\hline
\end{tabular}

Nota: Elaboración propia a partir de la aplicación del software SPSS 2019 versión 25. 
El nuevo modelo de atención imagine y su efecto en la satisfacción de los clientes del Banco Interbank de la tienda Comas 138, año 2015 - 2017

hipótesis alterna (H1), se concluye que hay alta satisfacción mientras que 174 valoran alta efecto significativo entre el nuevo modelo de atención Imagine y la empatía de los colaboradores. Véase Tabla 5.

Los clientes valoran la calidad de servicio. En la Tabla 6 se aprecia que los clientes están muy satisfechos respecto a la calidad de servicio, podemos notar que existe relación entre la calidad de servicio y el nuevo modelo de atención Imagine puesto que 64 clientes tienen muy satisfacción, de un total de 277 encuestados.

Finalmente se calculó el Chi-cuadrado de Pearson obteniendo como resultado $(\mathrm{X} 2=$ 200.700) y dado que es mayor que al chi-cuadrado crítico $(\mathrm{X} 2 \mathrm{cr}=21.02)$, rechazamos nuestra hipótesis nula (H0) y aprobamos la hipótesis alterna (H1), entonces se concluye que hay efecto significativo entre el nuevo modelo de atención Imagine y la calidad de servicio. Véase Tabla 7.

Tabla 4

Congruencia entre la empatía de los colaboradores y el nuevo modelo de atención Imagine

\begin{tabular}{|c|c|c|c|c|}
\hline Nuevo modelo de atención & $\begin{array}{c}\text { Consideración del } \\
\text { cliente }\end{array}$ & Frecuencia & $\begin{array}{l}\text { Porcentaje } \\
\text { válido }\end{array}$ & $\begin{array}{l}\text { Porcentaje } \\
\text { acumulado }\end{array}$ \\
\hline \multirow{6}{*}{ Empatía de los colaboradores } & Baja & 12 & 4.3 & 4.3 \\
\hline & Regular & 13 & 4.7 & 9.0 \\
\hline & Buena & 25 & 9.0 & 18.1 \\
\hline & Alta & 182 & 65.7 & 83.8 \\
\hline & Muy alta & 45 & 16.2 & 100.0 \\
\hline & Total & 277 & 100 & \\
\hline
\end{tabular}

Nota: Elaboración propia a partir de la aplicación del software SPSS 2019 versión 25.

Tabla 5

Chi-cuadrado entre la empatía de los colaboradores y el nuevo modelo de atención Imagine

\begin{tabular}{lccc} 
& Valor & Df & Significación Asintótica \\
Chi-cuadrado de Pearson & 229,387 & 12 & .000 \\
Razón de verosimilitud & 121,434 & 12 & .000 \\
Asociación lineal por lineal & 88,344 & 1 & .000 \\
Número de casos válidos & 277 & & \\
\hline
\end{tabular}

Nota: Elaboración propia a partir de la aplicación del software SPSS 2019 versión 25.

Tabla 6

Congruencia entre la calidad de servicio y el nuevo modelo de atención Imagine

\begin{tabular}{|c|c|c|c|c|}
\hline Nuevo modelo de atención & $\begin{array}{c}\text { Consideración del } \\
\text { cliente }\end{array}$ & Frecuencia & $\begin{array}{c}\text { Porcentaje } \\
\text { válido }\end{array}$ & $\begin{array}{l}\text { Porcentaje } \\
\text { acumulado }\end{array}$ \\
\hline \multirow{6}{*}{ Calidad de servicio } & Baja & 14 & 5.1 & 5.1 \\
\hline & Regular & 8 & 2.9 & 7.9 \\
\hline & Buena & 17 & 6.1 & 14.1 \\
\hline & Alta & 174 & 62.7 & 76.9 \\
\hline & Muy alta & 64 & 23.1 & 100.0 \\
\hline & Total & 277 & 100 & \\
\hline
\end{tabular}

Nota: Elaboración propia a partir de la aplicación del software SPSS 2019 versión 25.

Tabla 7

Chi-cuadrado entre la calidad de servicio y el nuevo modelo de atención Imagine

\begin{tabular}{lccc} 
& Valor & Df & Significación Asintótica \\
Chi-cuadrado de Pearson & 200,700 & 12 & .000 \\
Razón de verosimilitud & 120,143 & 12 & .000 \\
Asociación lineal por lineal & 108,173 & 1 & .000 \\
Número de casos válidos & 277 & & \\
\hline
\end{tabular}

Nota: Elaboración propia a partir de la aplicación del software SPSS 2019 versión 25. 
Se analizó los resultados obtenidos de las dimensiones de la variable independiente, los resultados indican una alta satisfacción de los clientes respecto a la capacidad de respuesta. En la Tabla 8 podemos notar que existe relación entre la capacidad de respuesta y el nuevo formato Imagine debido a que 49 clientes tienen muy alta satisfacción mientras que 175 valoran como alta, de un total de 277 encuestados.

Finalmente se calculó el Chi-cuadrado de Pearson obteniendo $(\mathrm{X} 2=285.925)$ y dado que es mayor que al chi-cuadrado crítico $(\mathrm{X} 2 \mathrm{cr}=$ 21.02), rechazamos nuestra hipótesis nula (H0) y aprobamos la hipótesis alterna (H1), debido a esto se concluye que hay efecto significativo entre el nuevo modelo de atención Imagine y la capacidad de respuesta. Véase Tabla 9.
El mobiliario es de alta calidad. En la Tabla 10 vemos que los clientes están muy satisfechos respecto a la moderna infraestructura inmobiliaria del banco, existe relación entre la moderna infraestructura inmobiliaria y el nuevo modelo de atención Imagine puesto que 83 clientes tienen muy alta satisfacción y 164 valoran alta satisfacción, de un total de 277 encuestados.

Finalmente se calculó el Chi-cuadrado de Pearson obteniendo $(X 2=316.542)$ y dado que es mayor que al chi-cuadrado crítico $(\mathrm{X} 2 \mathrm{cr}=$ 21.02), rechazamos nuestra hipótesis nula ( $\mathrm{HO})$ y aprobamos la hipótesis alterna (H1), se concluye que hay efecto significativo entre el nuevo modelo de atención Imagine y la moderna infraestructura inmobiliaria. Véase Tabla 11.

Tabla 8

Congruencia entre la capacidad de respuesta y el nuevo modelo de atención Imagine

\begin{tabular}{|c|c|c|c|c|}
\hline Nuevo modelo de atención & $\begin{array}{c}\text { Consideración del } \\
\text { cliente }\end{array}$ & Frecuencia & $\begin{array}{l}\text { Porcentaje } \\
\text { válido }\end{array}$ & $\begin{array}{l}\text { Porcentaje } \\
\text { Acumulado }\end{array}$ \\
\hline \multirow{6}{*}{ Capacidad de respuesta } & Baja & 10 & 3.6 & 3.6 \\
\hline & Regular & 15 & 5.4 & 9.0 \\
\hline & Buena & 28 & 10.1 & 19.1 \\
\hline & Alta & 175 & 63.2 & 82.3 \\
\hline & Muy alta & 49 & 17.7 & 100.0 \\
\hline & Total & 277 & 100 & \\
\hline
\end{tabular}

Nota: Elaboración propia a partir de la aplicación del software SPSS 2019 versión 25.

Tabla 9

Chi-cuadrado entre la capacidad de respuesta y el nuevo modelo de atención Imagine

\begin{tabular}{llll} 
& Valor & Df & Significación Asintótica \\
Chi-cuadrado de Pearson & 285,925 & 12 & .000 \\
Razón de verosimilitud & 188,214 & 12 & .000 \\
Asociación lineal por lineal & 151,221 & 1 & .000 \\
Número de casos válidos & 277 & & \\
\hline
\end{tabular}

Nota: Elaboración propia a partir de la aplicación del software SPSS 2019 versión 25.

Tabla 10

Congruencia entre la infraestructura inmobiliaria y el nuevo modelo de atención Imagine

\begin{tabular}{|c|c|c|c|c|}
\hline Nuevo modelo de atención & $\begin{array}{c}\text { Consideración del } \\
\text { cliente }\end{array}$ & Frecuencia & $\begin{array}{l}\text { Porcentaje } \\
\text { válido }\end{array}$ & $\begin{array}{l}\text { Porcentaje } \\
\text { Acumulado }\end{array}$ \\
\hline \multirow{6}{*}{ Infraestructura Inmobiliaria } & Baja & 13 & 4.7 & 4.7 \\
\hline & Regular & 3 & 1.1 & 5.8 \\
\hline & Buena & 14 & 5.1 & 10.8 \\
\hline & Alta & 164 & 59.2 & 70.0 \\
\hline & Muy alta & 83 & 30.0 & 100.0 \\
\hline & Total & 277 & 100 & \\
\hline
\end{tabular}

Nota: Elaboración propia a partir de la aplicación del software SPSS 2019 versión 25. 
El nuevo modelo de atención imagine y su efecto en la satisfacción de los clientes del Banco Interbank de la tienda Comas 138, año 2015 - 2017

En la Tabla 12 notamos que los clientes en la satisfacción del cliente del banco Interbank están muy satisfechos respecto a la publicidad utilizada por el banco Interbank, podemos resaltar que existe relación entre la publicidad y el nuevo modelo de atención Imagine puesto que 45 clientes tienen muy alta satisfacción mientras que 170 valoran alta satisfacción, de un total de 277 encuestados.

Finalmente se calculó el Chi-cuadrado de Pearson obteniendo como resultado $(\mathrm{X} 2=$ 143.984) y dado que es mayor que al chi-cuadrado crítico $(\mathrm{X} 2 \mathrm{cr}=21.02)$, rechazamos nuestra hipótesis nula (H0) y aprobamos la hipótesis alterna (H1), entonces se concluye que hay efecto significativo entre el nuevo modelo de atención Imagine y la publicidad. Véase Tabla 13.

\section{DISCUSIÓN}

La investigación demuestra que el nuevo modelo de atención Imagine tiene relación significativa tienda Comas 138. Se realizó la validez de la hipótesis general mediante las pruebas de chi-cuadrado y el promedio fue 48,491 por lo que afirmamos que existe relación entre las variables de esta hipótesis. Los beneficios de la implementación del modelo de atención Imagine no solo se enfocan en una moderna infraestructura, sino que también están orientadas a darle la facilidad a los clientes para que realicen sus transacciones, consultas e incluso reclamos de una manera más ágil.

Por este motivo los bancos centran su propósito y objetivos en la mejora continua en la calidad de servicio que por ello contar tecnología adecuada en para el desarrollo de sus operaciones, procesos claros, funcionarios con predisposición para la atención hacen que sean más valoradas por los clientes.

Tabla 11

Chi-cuadrado entre la infraestructura inmobiliaria y el nuevo modelo de atención Imagine

\begin{tabular}{lccc} 
& Valor & Df & Significación Asintótica \\
Chi-cuadrado de Pearson & 316,542 & 12 & .000 \\
Razón de verosimilitud & 180,804 & 12 & .000 \\
Asociación lineal por lineal & 144,101 & 1 & .000 \\
Número de casos válidos & 277 & & \\
\hline
\end{tabular}

Nota: Elaboración propia a partir de la aplicación del software SPSS 2019 versión 25.

Tabla 12

Congruencia entre la publicidad y el nuevo modelo de atención Imagine

\begin{tabular}{ccccc} 
Nuevo modelo de atención & $\begin{array}{c}\text { Consideración } \\
\text { del cliente }\end{array}$ & Frecuencia & $\begin{array}{c}\text { Porcentaje } \\
\text { válido }\end{array}$ & $\begin{array}{c}\text { Porcentaje } \\
\text { acumulado }\end{array}$ \\
\hline \multirow{3}{*}{ Publicidad } & Baja & 6 & 2.2 & 2.2 \\
& Regular & 11 & 4.0 & 6.1 \\
& Buena & 45 & 16.2 & 22.4 \\
& Alta & 170 & 61.4 & 83.8 \\
Muy alta & 45 & 16.2 & 100.0
\end{tabular}

Nota: Elaboración propia a partir de la aplicación del software SPSS 2019 versión 25.

Tabla 13

Chi-cuadrado entre la publicidad y el nuevo modelo de atención Imagine

\begin{tabular}{lccc} 
& Valor & Df & Significación Asintótica \\
Chi-cuadrado de Pearson & 143,984 & 12 & .000 \\
Razón de verosimilitud & 135,051 & 12 & .000 \\
Asociación lineal por lineal & 63,230 & 1 & .000 \\
Número de casos válidos & 277 & & \\
\hline
\end{tabular}

Nota: Elaboración propia a partir de la aplicación del software SPSS 2019 versión 25. 


\section{CONCLUSIONES}

El valor hallado de la fiabilidad del Alfa de Cronbach de las dimensiones en la variable dependiente nos muestra un valor de 0.97 y de las dimensiones de la variable independiente es 0.95 quiere decir que la correlación de los valores es muy buena. En este sentido se encontró que la dimensión transparencia de la información tiene un alto grado en la satisfacción de los clientes ya que el $53.1 \%$ y el $31.8 \%$ afirman tener una alta y buena satisfacción con esta dimensión respectivamente.

De igual forma la dimensión empatía de los colaboradores genera un alto grado de complacencia puesto que $16.2 \%$ considera una muy alta y el $65.7 \%$ afirma tener un alto grado de satisfacción. Asimismo, la dimensión calidad de servicio es la más valorada por los clientes en este sentido vemos que un $23.1 \%$ tiene muy alta satisfacción y un $62.8 \%$ alta complacencia con esta dimensión.

A pesar de que la capacidad de respuesta de los bancos no es muy valorada por los clientes, el $17.7 \%$ considera tener una alta satisfacción mientras que un $63.2 \%$ tiene alta satisfacción. De este modo las estadísticas demuestran un $30 \%$ tiene muy alta satisfacción con la dimensión moderna infraestructura inmobiliaria y el 59.2\% una alta satisfacción.
Por último, mencionamos que la dimensión publicidad es también valorada por los clientes ya que vemos un 16.2 muy satisfecho y el 61.4 altamente satisfecho.

\section{REFERENCIAS BIBLIOGRÁFICAS}

Franzmeier, J. (1994). Alcanzando la excelencia mediante el servicio al cliente. Madrid, España: Díaz de Santos.

Interbank. (2017). Interbank para mí. [Interbank Explora 5 razones por las que debes visitarlo]. Recuperado de https://interbank.pe/

Solorzano, B., \& Aceves, L. (2013). Importancia de la calidad de servicio al cliente para el funcionamiento de las empresas. El Buzón de Pacioli, 13(82), 4-13. Recuperado de https://www. itson.mx/publicaciones/pacioli/Documents/ no82/pacioli-82.pdf

Riveroll, E. (2016). Cómo deben los bancos y aseguradoras mirar al cliente. Forbes México. Recuperado de www.forbes.com.mx.

Uccelli, M. [Diario Gestión]. (2013). Interbank invertirá US\$ 60 millones en el rediseño innovador de sus agencias [Archivo de video]. Recuperado de https://www.youtube.com/watch?v=0FKlxN8f-Vs

Vieyra, E. (2014). Omnicanalidad: La nueva realidad del cliente bancario. Diario TI. Recuperado de https://diarioti.com/omnicanalidad-la-nueva-realidad-del-cliente-bancario/80343 